\section{Feasibility of endoscopic ultrasound-guided embolization of the splenic vein}

Hepatic encephalopathy is a complication that occurs when a large proportion of the mesenteric venous blood is shunted into the systemic circulation [1]. Spontaneous splenorenal or gastrorenal shunts may develop as portocaval circulation collaterals in patients with portal hypertension. Occlusion of spontaneous portal-systemic shunts by surgical or interventional radiological techniques is an effective treatment for intractable encephalopathy [2, $3]$. Selective embolization of the splenic vein was performed recently for untreatable portosystemic encephalopathy, with the application of metallic coils between a spontaneous splenorenal shunt and the superior mesenteric vein [4]. Ethylene vinyl alcohol (Enteryx) has been used for the embolization of vascular malformations [5]. In this study, we determined the feasibility of performing endoscopic ultrasound-guided splenic vein embolization (EUS-SVE) using Enteryx (Figure 1).

In a Yorkshire breed of pig, the splenic vein was identified by linear EUS (FG-36 UA; Pentax Precision Instruments Corp., Orangeburg, New York, USA), and a 22gauge fine-needle aspiration needle (Echo-3-22; Wilson-Cook, WinstonSalem, North Carolina, USA) was introduced into the splenic vein from the stomach. Under EUS guidance, $4 \mathrm{ml}$ of Enteryx was injected into the lumen of the splenic vein. The pressure in the splenic vein was measured before and after the injection. The animal was monitored for 7 days after the procedure and an upper abdominal contrast computed tomographic scan was performed on day 4 .

The splenic vein pressure increased from a mean of $0 \mathrm{mmHg}$ to a mean of $5 \mathrm{~mm} \mathrm{Hg}$ after EUS-SVE. Computed tomography on day 4 showed the Enteryx in the lumen of the splenic vein (Figure 2 ). Serum values of lipase, amylase, aspartate aminotransferase, alanine aminotransferase, creatinine, and blood urea nitrogen were all within normal limits. When the animal was sacrificed, a solid thrombus, $10 \mathrm{~mm} \times 5 \mathrm{~mm}$ in size, was visible inside

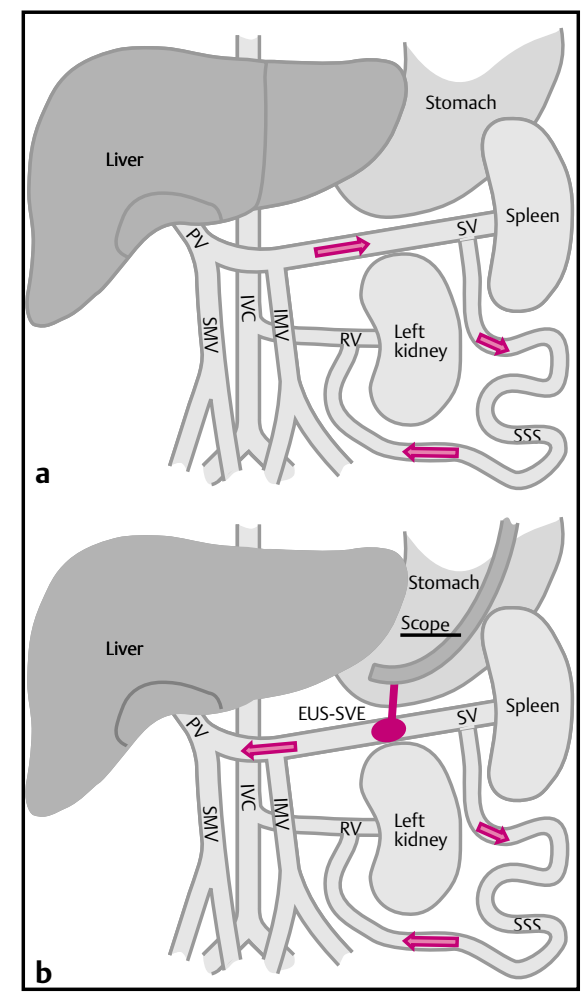

Figure 1 The technique of endoscopic ultrasound-guided splenic vein embolization, which leads to interruption of the hepatofugal flow from the splenic vein to the splenorenal shunt, the left renal vein, and the inferior vena cava, while maintaining drainage of the splenic vein through the splenorenal shunt. The diagram shows the venous blood flow before (a) and after (b) the procedure. The patient's encephalopathy subsides as a result of the reversal of the mesenteric venous bypass.

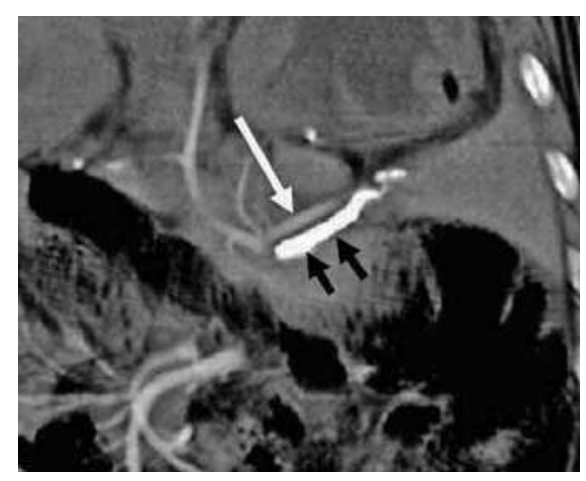

Figure 2 Contrast-enhanced, coronal, threedimensional, multiplanar reformatted computed tomographic image showing Enteryx in the splenic vein (black arrows). The splenic artery is illustrated by a white arrow.

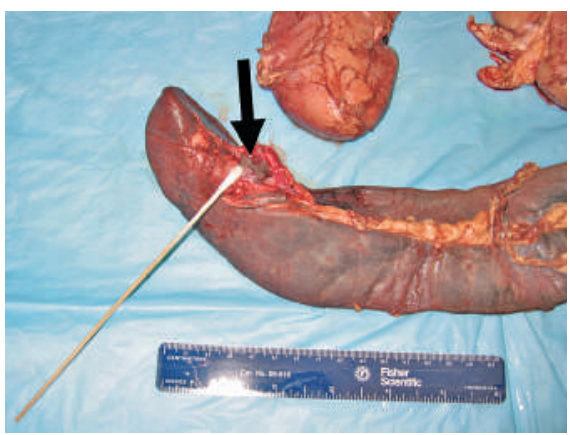

Figure 3 The solid thrombus, $10 \mathrm{~mm} \times 5 \mathrm{~mm}$ in size, that was visible in the splenic vein at postmortem (black arrow).

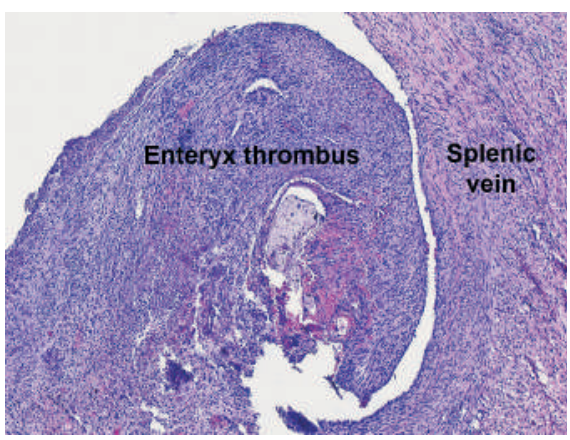

Figure 4 Histological view showing an Enter$y x$ thrombus in the splenic vein (hematoxylin $\&$ eosin stain, $\times 50$ magnification).

the splenic vein (Figure 3), and this was confirmed by histology (Figure 4).

EUS-SVE using Enteryx appears to be a feasible method for embolizing the splenic vein, and was well tolerated during a 1-week observational period. This technique could be considered as an alternative to direct shunt occlusion by surgery or interventional radiology.

Endoscopy_UCTN_Code_TTT_1AS_2AC 
K. Matthes' ${ }^{1}$ D. Sahani ${ }^{2}$, N. S. Holalkere ${ }^{2}$, M. Mino-Kenudson ${ }^{3}$, W. R. Brugge ${ }^{1}$

${ }^{1}$ Gastrointestinal Unit, Massachusetts General Hospital, Harvard Medical School, Boston, Massachusetts, USA

2 Department of Radiology, Massachusetts General Hospital, Harvard Medical School, Boston, Massachusetts, USA

${ }^{3}$ Gastrointestinal Pathology Service, Department of Pathology, Massachusetts General Hospital, Harvard Medical School, Boston, Massachusetts, USA.
References

${ }^{1}$ Takashi M, Igarashi M, Hino Set al. Portal hemodynamics in chronic portal-systemic encephalopathy: angiographic study in seven cases. J Hepatol 1985; 1: 467-476

${ }^{2}$ Hsieh JS, Wang JY, Huang CJet al. Effect of spontaneous portosystemic shunts on hemorrhage from esophagogastric varices. World J Surg 2004; 28: 23-28

${ }^{3}$ Miyamoto Y, Oho K, Kumamoto Met al. Balloon-occluded retrograde transvenous obliteration improves liver function in patients with cirrhosis and portal hypertension. J Gastroenterol Hepatol 2003; 18: 934-942

${ }^{4}$ Mezawa S, Homma H, Akiyama Tet al. Selective embolization of the splenic vein in patients with hepatic encephalopathy and splenorenal shunt. J Vasc Interv Radiol 2004; 15: 1475 - 1481

${ }^{5}$ Hamada J, Kai Y, Morioka Met al. A nonadhesive liquid embolic agent composed of ethylene vinyl alcohol copolymer and ethanol mixture for the treatment of cerebral arteriovenous malformations: experimental study. J Neurosurg 2002; 97: 889-895
Corresponding author

\section{W. R. Brugge, M.D.}

\section{Fruit Street}

Blake 4

Boston

Massachusetts A 02114

USA

Fax: $\quad+1-617-724-5997$

Email: wbrugge@partners.org 